\title{
Roentgenographic Features in Antibiotic-Associated Pseudomembranous Colitis
}

\author{
Kyung J. Cho, M.D., Y. M. Ting, M.D., Vincent P. Chuang, M.D., Inkyu Lee, M.D. \\ From the Department of Radiology, University of Michigan, Ann Arbor \\ and Wayne County General Hospital, Eloise, Michigan 48132
}

\section{Case Presentations}

Case 1. A 62-year-old woman with diabetes was admitted because of an infected toe. Physical examination was normal except for an ulceration of the left 4th toe. Routine laboratory studies revealed only elevated blood sugar and moderate ketonuria. A roentgenogram of the toe revealed osteomyelitis. The infection improved with clindamycin therapy $(300 \mathrm{mg}$ IV, $t: d)$, and the toe was amputated on the 6 th day of admission. Five days after initiation of antibiotic therapy the patient developed severe diarrhea and emesis. A barium enema showed mucosal irregularity throughout the entire colon with areas of dissection of barium under pseudomembranes (Figures 1A, 1B). Sigmoidoscopy and biopsy confirmed the diagnosis of pseudomembranous colitis. The patient developed pneumonia and myocardial infarction, and died.

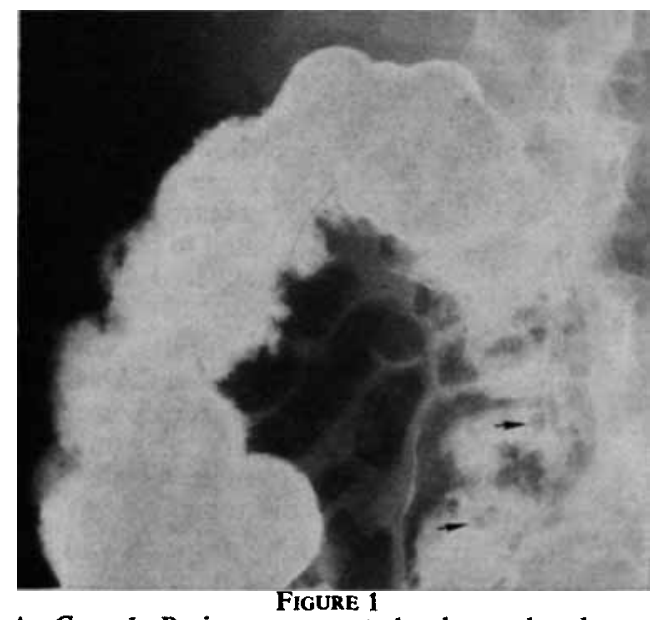

A. Case 1. Barium enema study shows the shaggy contour of the margins of ascending and transverse colons, resembling diffuse ulceration. Note multiple rounded or ovoid defects simulating polyps in the proximal air-filled transverse colon (arrows).

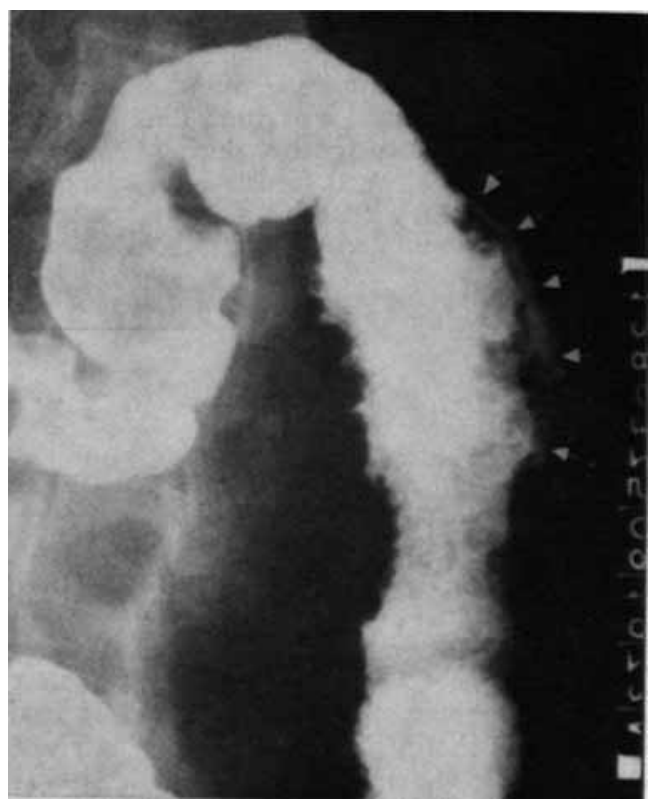

Figure 1

B. Detailed view of splenic flexure of the colon shows barium dissection underneath pseudomembranes (arrows).

Case 2. A 59-year-old man with chronic renal failure underwent nephrectomy and resection of a previous ileal loop. Post-operatively, he developed abdominal distention and rectal bleeding after four days of treatment with clindamycin and gentamycin. Serial roentgenograms of the abdomen revealed thickening of the haustra and intramural gas in the cecum and ascending colon (Figure 2). Sigmoidoscopic findings confirmed the diagnosis of pseudomembranous colitis. The biopsy revealed mucopurulent material mixed with neutrophils. Stool cultures were non-specific. The patient continued to bleed from the colon. An inferior mesenteric 


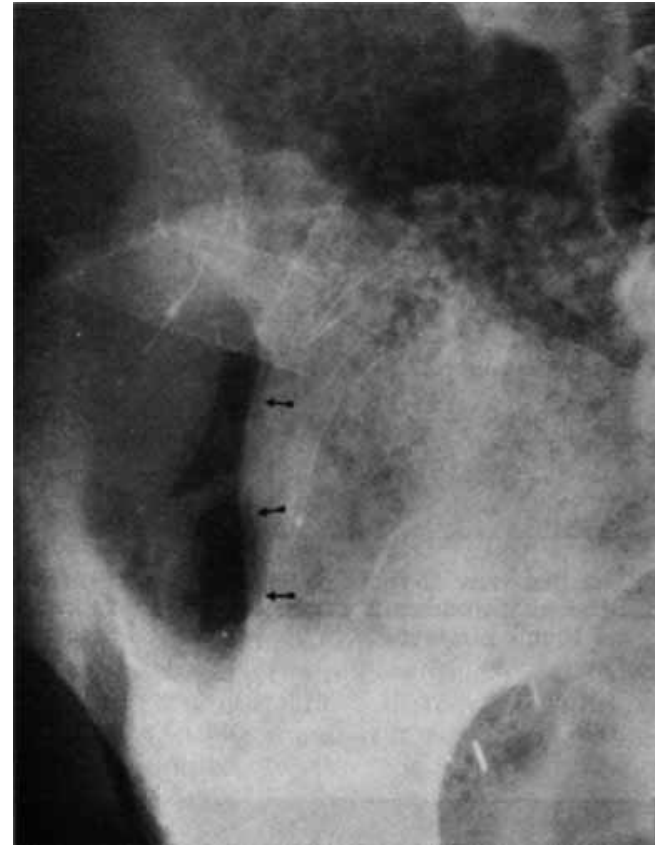

Figure 2

Case 2. Radiograph of the abdomen demonstrates gas in the wall of the cecum and ascending colon (arrows).

angiogram revealed active bleeding from the proximal transverse colon (Figure 3 ). Although pitressin was infused, the patient continued to bleed, requiring 50 units of blood. He finally underwent ileostomy and had a cardiac arrest. At autopsy, pseudomembranes involved the entire colon.

Case 3. A 32-year-old man with chronic pyelonephritis and nephrosclerosis underwent bilateral nephrectomy and renal transplantation on three occasions with rejection in each instance. He had been on long-term hemodialysis. Two weeks before death he developed abdominal tenderness, intermittent bloody diarrhea and fever. He was treated with Vancomycin, Colymycin and Ampicillin for four weeks before the onset of bloody diarrhea. A barium enema showed marked mucosal irregularity due to submucosal edema (Figures 4A, 4B). At autopsy pseudomembranous colitis involved the entire colon.

Case 4. A 50-year-old man was admitted because of ten days of bloody diarrhea. He had received erythromycin for two weeks before the

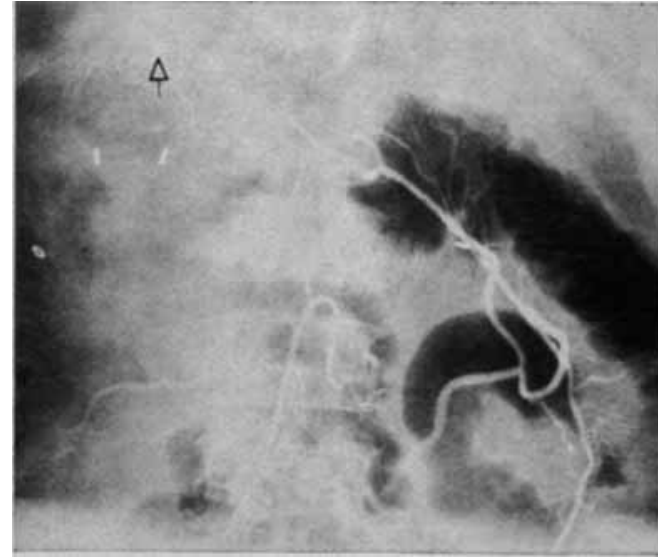

A. Arterial Phase

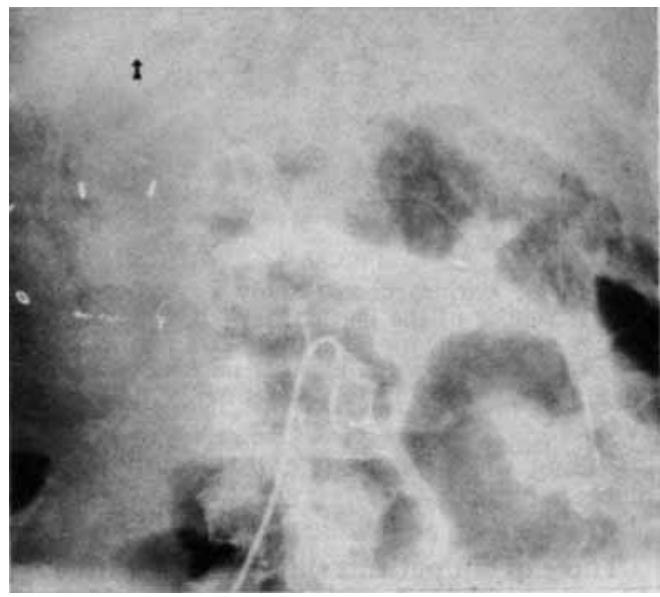

B. Venous Phase

Figure 3

Case 2. Inferior mesenteric arteriogram.

There is active arterial bleeding in the proximal transverse colon (arrow). Selective intra-arterial infusion of Pitressin into both superior and inferior mesenteric arteries failed to control the bleeding.

onset of diarrhea because of chronic sinusitis. Pseudomembranes were seen at sigmoidoscopy. A barium enema showed mild mucosal irregularity in the descending and sigmoid colons (Figure 5), which returned to normal following an antidiarrheal regimen and Azulphidine.

\section{Discussion}

Plain abdominal radiographic findings in pseudomembranous colitis are nonspecific, 
Kyung J. Cho, Y. M. Ting, Vincent P. Chuang And Inkyu Lee

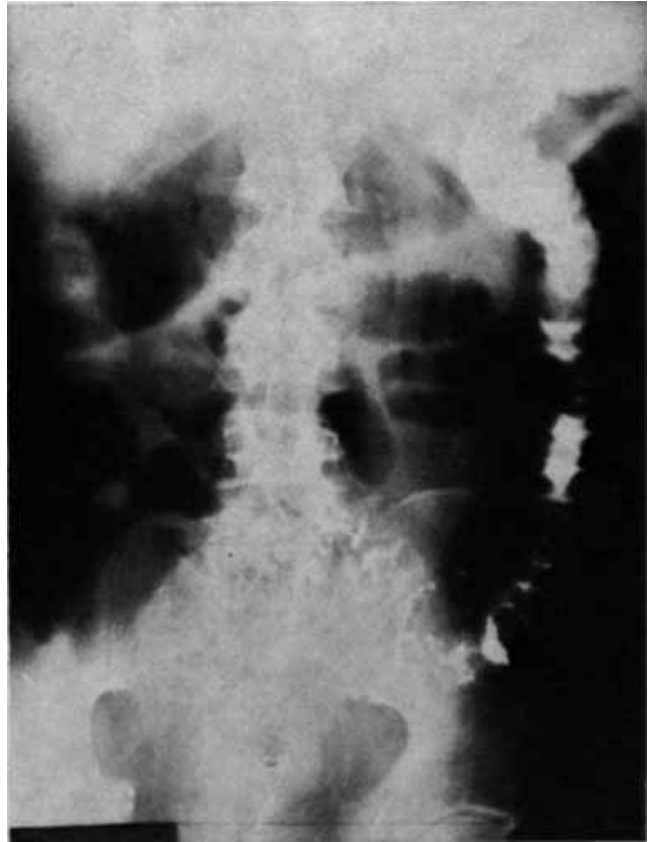

FIGURe 4

A. Case 3. Barium enema study demonstrated the shaggy contour of the barium filled sigmoid and descending colon. The air-filled transverse and ascending colons show the haustral thickening.

including disproportionate distension of segments of the intestine, which may simulate obstruction or adynamic ileus. ${ }^{3}$

Three of our patients showed non-obstructive dilatation of bowel, with an adynamic ileus gas pattern, and of these one had intramural gas in the cecum.

Barium enema findings have been reported as being nonspecific and indistinguishable from other types of colitis. ${ }^{10}$ Stanley described a radiographic spectrum ranging from the nonspecific changes to the diagnostic findings. ${ }^{12}$

All patients in this series had abnormal radiographic findings. Barium enema showed a wide spectrum of roentgenographic features, ranging from mild, focal areas of mucosal irregularity to widespread involvement of the entire colon with multiple confluent pseudomembranes. The mucosal irregularity at barium enema is caused by plaque-like pseudomembranes studding the mucosal surface. These vary in size and appear as oval or rounded filling defects when seen en face, mimicking pseudopolyps on air contrast

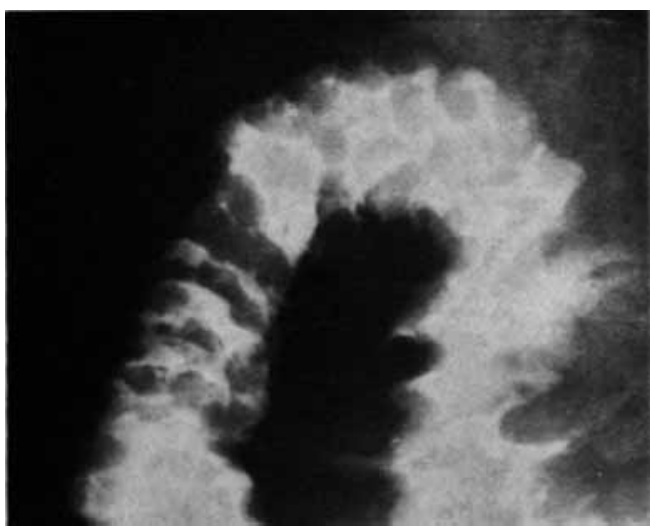

B. Detailed view of the sigmoid colon shows plaquelike lesions with areas of confluent pseudomembranes, and thumb printing due to the submucosal edema.

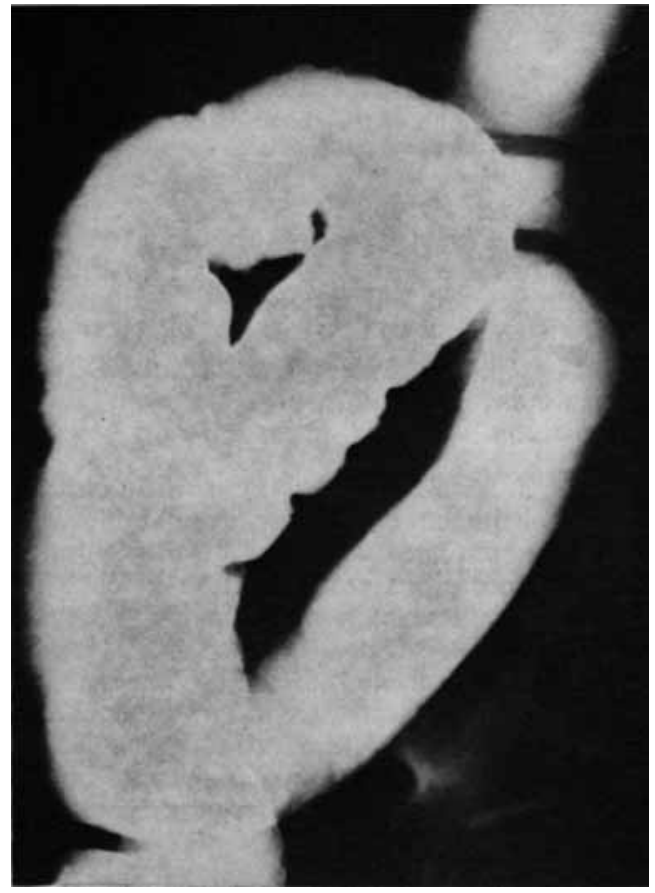

Figure 5

Case 4. There is mucosal irregularity along the sigmoid colon, which could well be interpreted as mucous secretion. The colon returned to normal after seven weeks treatment.

Australasian Radiology, Vol. XX, No. I, March, 1976 


\section{Roentgenographic Features in Antiobiotic-Associated Pseudomembranous Colitis}

studies (Figure 1A). In profile, they have a shaggy contour, simulating ulceration (Figure 1B). Nonspecific haustral thickening and thumbprinting due to submucosal edema similar to that seen in other inflammatory bowel disease also occur. Multiple areas of barium dissection under the pseudomembranes and possibly even under the mucosa, in addition to extensive mucosal irregularity as seen in Case 1, are considered specific for this disease. Gas was present in the wall of the cecum in Case 2. Intramural gas is often seen in ischemic colitis.

The longitudinal dissection of barium under the pseudomembranes observed in one patient has been reported in one other patient. ${ }^{7}$ This dissection parallels the bowel lumen, probably running between the pseudomembranes and mucosa. It can be distinguished from the intramural fistulous tract, of granulomatous colitis, which shows multiple transverse fissures producing a stepladder configuration. ${ }^{6}$ The roentgenographic abnormalities in regional enteritis are usually localized and segmental in distribution in contrast to the widespread colonic involvement caused by pseudomembranes.

Pseudomembranous colitis can generally be confirmed by observing pseudomembranes at sigmoidoscopy and biopsy. They appear as creamy, elevated patches of varying size, adherent to the mucosal surface. The intervening mucosa may be normal or erythematous. Ulceration is unusual but may occur in the late stage of the disease when pseudomembranes slough off. Histologically, the pseudomembranes consist of fibrin, mucous material and inflammatory cells.

It is emphasized that massive colonic bleeding from pseudomembranous colitis may not respond to the selective intra-arterial infusion of vasopressin.

\section{SUMMARY}

Four patients with pseudomembranous colitis, related to antibiotic therapy, had a wide spectrum of radiographic features. These ranged from mild, focal areas of mucosal irregularity to widespread involvement of the colon. The extensive mucosal irregularity at barium enema is caused by plaque-like elevated pseudomembranes studding the mucosal surface and appears to be a specific radiographic finding. Dissection of barium under the pseudo-membranes and intramural gas was observed in these patients. Case 2 had a complicated, massive colonic bleeding, unresponsive to pitressin infusion.

Although pseudomembranous colitis is a well established pathological entity, the precise pathogenesis is still uncertain. Recently, an increasing number of patients have been described with antibiotic-associated pseudomembranous colitis. 1.11 .12 The disease is also known to occur as a complication of colonic obstruction, staphylococcal overgrowth, following abdominal surgery and in generalized debilitating diseases, such as uremia, lymphoma and leukemia. ${ }^{4}, 5,8,8$

We have examined four patients with pseudomembranous colitis associated with antibiotic therapy. A wide range of roentgenographic abnormalities was observed in these patients.

\section{REFERENCES}

${ }^{1}$ Benner, E. J., and Telknan, W. H. (1970): "Pseudomembranous colitis as a sexuel to oral Lincomycin Therapy." Amer. J. Gastroenterol, 54, 55.

${ }^{2}$ Cohen, L. E., McNeill, C. J., and Wells, R. F. (1973): "Clindamycin-associated colitis." J.A.M.A., 223, 1379.

${ }^{3}$ Feinberg, S. B. (1960): "Roentgen findings in severe pseudomembranous enterocolitis." Radiology, 74, 778.

'Goulston, S. J. M., and McGovern, V. J. (1965): "Pseudomembranous colitis." Gut, 6, 207.

${ }^{5}$ Hale, H. W. Jr., and Cosgriff, J. H. Jr. (1957): "Pseudomembranous enterocolitis." Amer. $J$. Surg., 94, 710.

'Marshak, R. H., Janowitz, H. D., and Present, D. H. (1970): "Granulamatous colitis in association with diverticula." The New England Journal of Medicine. 283, 1080.

'Owen, G. M. (1957): "Role of antibiotics in pseudomembranous enteritis." J. Iowa Med. Soc., 47, 237.

"Pettet, J. D., Baggenstoss, A. H., Dearing, W. H., and Judd, E. S. Jr. (1954): "Post-operative pseudomembranous enterocolitis." Surg. Gynec. Obstet.. $98,546$.

${ }^{9}$ Prohaska, J. V. (1959): "Pseudomembranous enterocolitis. The experimental induction of the disease with staphyloccus aureus and its enterotoxin." Arch. Surg. (Chicago). 79, 197.

"Sherbon, K. J. (1972): "Radiology in pseudomembranous colitis." A ust. Radiol. I6, 66.

${ }^{11}$ Shimkin, P. M., and Link, R. J. (1973): "Pseudomembranous colitis: a consideration in the barium enema differential diagnosis of acute generalized ulcerative colitis." Brit. J. Radiol., 46, 437.

${ }^{1 n}$ Stanley, R. J., Melson, G. L., and Tedesco. F. J. (1974): "The spectrum of radiographic findings in antibiotic-related pseudomembranous colitis." Radiology 111, 519. 